\title{
A peptide immunoaffinity LC-MS/MS strategy for quantifying the GPCR protein, S1PR1 in human colon biopsies
}

\author{
Hongwei Zhang*iD, Eugene Ciccimaro, Jacob Zalaznick, Bogdan G Sleczka, Laurence \\ Menard, Timothy V Olah \& Petia Shipkova \\ Research \& Development, Bristol Myers Squibb, Princeton, NJ 08543, USA \\ *Author for correspondence: Tel.: +1 609-252-6797; hongwei.zhang@bms.com
}

Background: S1PR1, a G protein-coupled receptor (GPCR) protein, is a therapeutic target for treatment of autoimmune diseases. As a potential biomarker for drug effect and patient stratification, it is of great significance to measure it in biological samples. However, due to the hydrophobic nature of S1PR1 and the difficulties in extraction and solubilization, as well as low expression levels, quantitative determination of S1PR1 remains challenging. Results: In this work, a peptide immunoaffinity LC-MS/MS method was developed to quantify S1PR1 in biopsy-sized colon samples with an LLOQ of 7.81 pM. Conclusion: Peptide immunoaffinity LC-MS/MS based strategy has achieved the desired sensitivity for low abundance S1PR1, and the same strategy could be applied to quantify S1PR1 in multiple species and other GPCR proteins.

First draft submitted: 27 May 2020; Accepted for publication: 12 August 2020; Published online:

18 September 2020

Keywords: GPCR • LC-MS/MS • multi-transmembrane protein • peptide immunoaffinity enrichment • protein biomarkers • quantitation $\bullet$ S1PR1

Sphingosine-1-phsophate (S1P) is a lysophospholipid mediator that evokes a variety of cellular responses such as proliferation, migration, survival, angiogenesis, inflammation and lymphocyte egress by stimulation of five members of G-protein coupled receptors S1PR1, R2, R3, R4, R5, of which the S1PR1 receptor is most extensively investigated [1,2]. S1PR1 receptors are expressed in many cell types including lymphocytes and endothelial cells [3]. S1P induces sustained S1PR1 signaling with receptor recycling. Targeting S1P/ S1PR1 pathway has the potential to be an effective therapy for broad indications including lymphocyte-mediated autoimmune diseases [3-5]. Synthetic S1PR1 agonists induce transient S1PR1 signaling, internalization and degradation, and work as functional antagonists to inhibit S1P induced cell migration $[4,6]$. As a result, lymphocytes are sequestered in the thymus and secondary lymphoid organs without entering circulation. S1PR1 levels could be a potential biomarker for drug effect and patient stratification, and a quantitative assay suitable for colon biopsy samples was needed. To our knowledge, there have been no absolute quantitative assays for S1PR1 concentration levels in biological samples reported.

S1PR1 belongs to the family of G protein-coupled receptors (GPCRs), which are also known as seventransmembrane domain receptors. GPCRs share common structure, with an extracellular N-terminus, 7transmembrane helices linked by three extracellular (EL1-3) loops and three intracellular loops (IL1-3), and an intracellular C-terminus (Figure 1). The seven-transmembrane helices are arranged in a structurally conserved bundle and highly hydrophobic. GPCRs are the largest and most diverse group of membrane receptors with hundreds of different members. As cell-surface signal transmission receptors, they mediate most of the physiological responses to growth factors, hormones, neurotransmitters and environmental stimulants [7] and transduce an extracellular signal to an intracellular response through their transmembrane. Their importance is demonstrated by the fact that more than one-third of the currently marketed therapeutic medicines target more than 100 unique GPCRs for a broad spectrum of indications including cancer, infection, inflammation and cardiovascular disease etc. [8-10]. Furthermore, approximately $20 \%$ of new agents currently in clinical trials target more than 60 novel GPCRs [9]. Due to recent advances in structural biology, pharmacology and biotechnology, GPCR drug discovery 


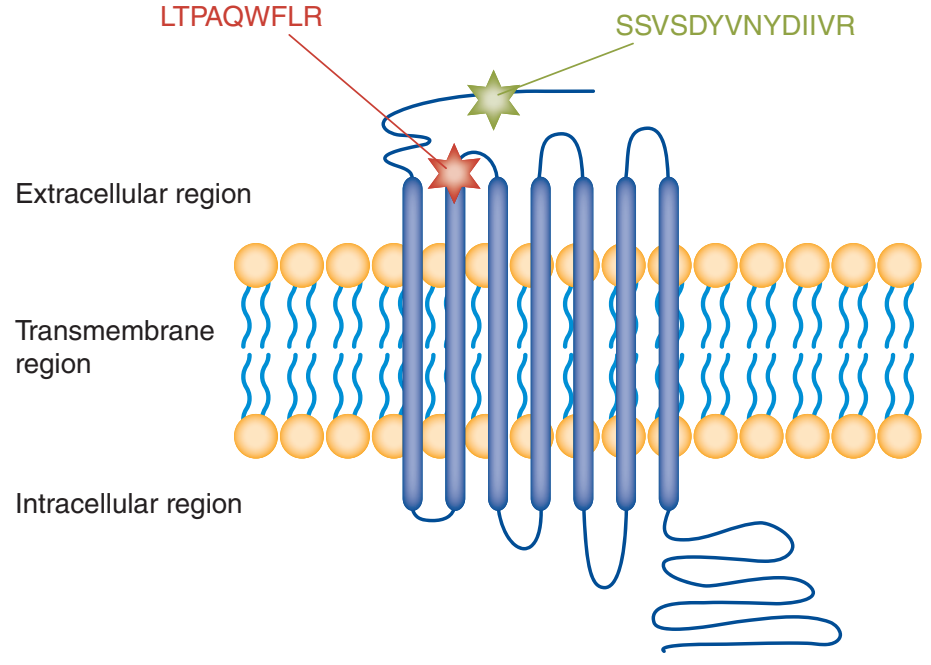

Figure 1. Structural biology of the S1PR1 receptor.

has been growing fast, as demonstrated by numerous emerging novel drug targets. Bioanalytical strategies to measure GPCR proteins are of significant interest for drug discovery and development. However, due to their hydrophobic nature and intrinsic difficulties of extraction and solubilization, as well as low expression levels, direct quantitative determination of GPCR proteins remains challenging. The measurement of mRNA expression is usually used for prediction of relative expressions of GPCRs in cells and tissues [11]. For development of immuno-based assays, several obstacles have been encountered around the generation of specific antibodies against the GPCR proteins. First, detergent based solubilization of the GPCR immunogen causes major conformational changes but even subtle changes in conformation could affect the access of antibodies to the epitopes [12]. Second, since the epitopes are made up of small discontinuous extracellular segments, the chance for producing a strong immune response is significantly weakened. As a result, it is extremely difficult to generate specific and high-affinity antibodies. The dominant issue for GPCR antibody is lack of selectivity and a high level of cross-reactivity with other GPCRs due to the similarity in their protein sequences [13]. Third, it is difficult to obtain sufficient material for immunization due to low expression levels and solubility. It is also difficult to express them recombinantly. Given these difficulties, it is challenging to develop anti-protein based assays for GPCR protein quantitation.

In this work, our strategy is peptide immunoaffinity enrichment followed by LC-MS/MS detection. It bypasses most of those obstacles encountered for anti-protein immunoaffinity enrichment. Peptide immunoaffinity enrichment specifically captures the peptides of interest from complex biological matrices. Mass spectrometry has evolved as a powerful technology platform for specific detection of proteins from peptide level based on their mass-tocharge ratio and CID induced fragments [14-19]. Coupling peptide immunoaffinity enrichment with LC-MS/MS detection enables sufficient sensitivity and specificity for low-abundance endogenous proteins [20-26] and avoids the restrictions associated with traditional sandwich immunoassays. The LC-MS/MS approach could substantially saves the time and cost involved in screening for a pair of antibodies [27,28]. Peptide immunoaffinity LC-MS/MS assays, also termed stable isotope standards and capture by anti-peptide antibodies (SISCAPA) as first reported by Anderson et al. [29], have been employed for protein biomarker quantitation as a complementary bioanalytical strategy to avoid the limitations inherent to immunoassays, such as potential interference from endogenous immunoglobulins [22]. Whiteaker et al. demonstrated the application of a multiplexed immuno-MRM assay targeting phospho-signaling [30,31]. In this work, we developed a peptide immunoaffinity LC-MS/MS assay for quantitative determination of a GPCR protein, S1PR1 in small biopsy-sized human colon tissue samples. The sample size was limited to a small-sized biopsy, since the ultimate goal was to apply this approach to clinical studies where only colon biopsy samples are collected by endoscopy. This paper details the challenges, strategies and method optimization for sample extraction, peptide selection and immunocapture to achieve the desired sensitivity and selectivity. Since all the GPCR proteins share similar structures, from bioanalytical perspective, it is very important to understand these challenges and establish bioanalytical strategies for assay development for GPCR protein quantitation. 


\begin{tabular}{|c|c|c|c|c|}
\hline Peptide & Precursor ion & Product ion & DP & CE \\
\hline LTPAQWFLR.+2y7.light & 566.3 & 917.5 & 72.4 & 28 \\
\hline LTPAQWFLR.+2y6.light & 566.3 & 820.4 & 72.4 & 32 \\
\hline LTPAQWFLR.+2y7.heavy & 571.3 & 927.5 & 72.4 & 28 \\
\hline LTPAQWFLR.+2y6.heavy & 571.3 & 830.5 & 72.4 & 32 \\
\hline SSVSDYVNYDIIVR.+2y8.light & 815.4 & 991.6 & 90.6 & 37 \\
\hline SSVSDYVNYDIIVR.+2y7.light & 815.4 & 892.5 & 90.6 & 38 \\
\hline SSVSDYVNYDIIVR.+2y8.heavy & 820.4 & 1001.6 & 90.6 & 37 \\
\hline SSVSDYVNYDIIVR.+2y7.heavy & 820.4 & 902.5 & 90.6 & 38 \\
\hline
\end{tabular}

\section{Experimental}

\section{Materials \& reagents}

Recombinant human S1PR1 (H00001901-G01) containing 382 amino acids with molecular weight of $42.8 \mathrm{kDa}$ was obtained from Abnova. It is a human S1PR1 full-length recombinant protein without tag. Anti-peptide antibodies, anti-LTPAQWFLR and anti-SSVSDYVNYDIIVR, which are rabbit polyclonal antibodies, were custom-made at GenScript. Magnetic tosylactivated beads (Invitrogen Dynabeads M-280), magnetic protein G beads (Invitrogen Dynabeads), Pierce DTT (Dithiothreitol) and Pierce iodoacetamide for single-use, HALT protease inhibitor cocktail and protein extraction detergents including T-PER, RIPA, CHAPS, NP-40, Triton X-100, Brij ${ }^{\circledR}-35$, were obtained from Thermo Fisher Scientific (CA, USA). Trypsin Gold, sequencing grade-modified trypsin was obtained from Promega Corporation. Bovine serum albumin (BSA), phosphate buffered saline (PBS) and bovine pancreatic trypsin were purchased from Sigma-Aldrich. Stable-isotope-labeled (SIL) analogs of the surrogate peptides, SIL-LTPAQWFLR and SIL-SSVSDYVNYDIIVR, which were labeled with heavy arginine with a mass shift of +10 , were custom-synthesized at New England Peptide. PBST buffer was prepared by adding $0.05 \%$ Tween-20 into PBS. Zwittergent 3-12 detergent was obtained from Emdmillipore. Human colon samples were purchased from Analytical Biological Services Inc.

\section{Instrumentation \& LC-MS/MS conditions}

A quadrupole Orbitrap (Q Exactive, Thermo Fisher Scientific) operated in positive ESI mode was coupled to a LC system for surrogate peptide identification from recombinant human S1PR1 tryptic digest. Data-dependent acquisition (DDA) was performed to collect MS1 full scan and MS/MS spectra. Proteome Discoverer software was used to process data for peptide identification. Skyline was employed for building multiple reaction monitoring (MRM) acquisition methods for the collision energy (CE) optimization. AB SCIEX Triple Quad 6500 system (ABI/MDS Sciex, Concord, ON, Canada) equipped with a Turbolonspray source in positive MRM mode was used for detection of the surrogate peptides. Q1 and Q3 were operated in unit resolution. Quantitative analysis was conducted by using Analyst 1.6 software (AB Sciex). Two surrogate peptides, LTPAQWFLR and SSVSDYVNYDIIVR were identified and two MRM transitions were monitored for each peptide. The precursor and product ions of MRM transitions, their optimized collision energy and their default declustering potential predicted from Skyline, for the surrogate peptides and corresponding SIL peptides are listed in Table 1. A Waters Acquity UPLC I-Class system was coupled to the mass spectrometer for LC separation. The binary gradient was applied to a $2.1 \times 50 \mathrm{~mm}$,

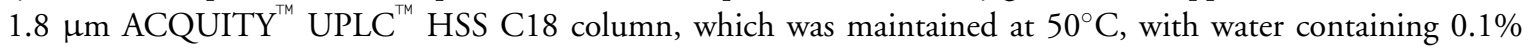
formic acid (A) and acetonitrile containing $0.1 \%$ formic acid (B) mobile phases at a flow rate of $800 \mu \mathrm{l} / \mathrm{min}$. The gradient started at $10 \% \mathrm{~B}$, and held for $0.5 \mathrm{~min}$. Then the gradient was ramped up linearly from $10 \% \mathrm{~B}$ to $35 \%$ B over $2.5 \mathrm{~min}$ followed by washing with $95 \% \mathrm{~B}$ and column equilibration. The chromatographic run time was $5 \mathrm{~min}$.

\section{Standard \& quality control}

The stock solution of S1PR1 recombinant protein has a concentration of $0.02 \mu \mathrm{g} / \mu \mathrm{l}$ in $25 \mathrm{mM}$ Tris- $\mathrm{HCl}$ buffer of $\mathrm{pH} 8.0$ containing 2\% glycerol. It was diluted to $100 \mathrm{nM}$ in PBS buffer containing $0.05 \%$ zwittergent and stored at $-80^{\circ} \mathrm{C}$. Calibration standards (STD) of S1PR1 recombinant protein at 7.81, 15.6, 31.3, 62.5, 125,250 and 


\begin{tabular}{|c|c|c|}
\hline Extraction buffer & Composition & lonicity \\
\hline PBS & Phosphates and sodium salts & Isotonic \\
\hline T-PER & Proprietary detergent in $25 \mathrm{mM}$ bicine, $150 \mathrm{mM} \mathrm{NaCl}$ & Zwitterionic \\
\hline $5 \%$ CHAPS in PBS & 3-[(3-cholamidopropyl)dimethylammonio]-1-propanesulfonate & Zwitterionic \\
\hline RIPA & $25 \mathrm{mM}$ Tris॰ $\mathrm{HCl} \mathrm{pH}$ 7.6, $150 \mathrm{mM} \mathrm{NaCl}, 1 \% \mathrm{NP}-40,1 \%$ sodium deoxycholate, $0.1 \%$ SDS & $\begin{array}{l}\text { Nonionic/ } \\
\text { anionic }\end{array}$ \\
\hline $5 \% \mathrm{NP}-40$ in PBS & $\begin{array}{l}10 \% \text { NP-40 (nonyl phenoxypolyethoxylethanol) in Ultrapure Water } \\
\text { Diluted 1:2 in PBS }\end{array}$ & Nonionic \\
\hline $1 \%$ Triton $\mathrm{X}-100$ in PBS & $\begin{array}{l}10 \% \text { Triton } X-100 \text { in Ultrapure Water } \\
\text { Diluted } 1: 10 \text { in PBS }\end{array}$ & Nonionic \\
\hline $5 \%$ Triton $\mathrm{X}-100$ in PBS & $\begin{array}{l}10 \% \text { Triton X-100 in Ultrapure Water } \\
\text { Diluted } 1: 2 \text { in PBS }\end{array}$ & Nonionic \\
\hline $5 \% \mathrm{Brij}^{\circledR}-35$ in PBS & $\begin{array}{l}10 \% \text { Brij }^{\circledR}-35 \text { in Ultrapure Water } \\
\text { Diluted } 1: 2 \text { in PBS } \\
\text { Contains ethoxylated lauryl alcohol }\end{array}$ & Nonionic \\
\hline
\end{tabular}

$500 \mathrm{pM}$ were prepared by serial dilution in lysis buffer containing $0.1 \%$ BSA and 1\% HALT protease inhibitor cocktail. With the same stock solution, quality controls (QCs) of S1PR1 recombinant protein at 15.6, 31.3, 62.5, 125, 250 and 500 pM were prepared separately and three replicates for each level were analyzed.

The stock solution of SIL-LTPAQWFLR was prepared in $50 \mathrm{mM}$ ammonium bicarbonate buffer containing $0.05 \%$ zwittergent at a concentration of $1 \mathrm{mM}$ and stored at $-80^{\circ} \mathrm{C}$. The calibration standards of heavy surrogate peptide, SIL -LTPAQWFLR at 7.81, 15.6, 31.3, 62.5, 125, 250 and 500 pM were prepared by serial dilution in $50 \mathrm{mM}$ ammonium bicarbonate buffer containing $0.05 \%$ zwittergent. With the same stock solution, QCs of heavy surrogate peptide, SIL -LTPAQWFLR at 15.6, 31.3, 62.5, 125, 250 and 500 pM were prepared separately and three replicates for each level were analyzed.

\section{Tissue colon pulverization, homogenization \& total protein measurement Tissue homogenization}

Colon tissue sample was pulverized into powder in TT1 tissue tube (Covaris, Inc.) by using CryoPREP ${ }^{\mathrm{TM}}$ Impactors (Covaris, Inc.) right after it was dipped in liquid nitrogen. Aliquots of the pulverized colon $(\sim 5 \mathrm{mg})$ were put into $0.5 \mathrm{ml} \mathrm{CK} 14$ homogenization tubes containing $1.4 \mathrm{~mm}$ ceramic (zirconium oxide) beads (Bertin Instruments). A volume of $200 \mu \mathrm{l}$ of T-PER buffer with $1 \%$ HALT protease inhibitor cocktail was added to the samples. The samples were then homogenized at $4^{\circ} \mathrm{C}$ using two cycles of $30 \mathrm{sec}$ at $6500 \mathrm{rpm}$ with pauses of 30 sec between each cycle by using the Precellys Evolution Homogenizer with Cryolys Evolution (Bertin Instruments). Thus, total processing time for lysis is $2 \mathrm{~min}$. Following centrifugation at $14,000 \times \mathrm{g}$ for $10 \mathrm{~min}$ at $4^{\circ} \mathrm{C}$, the supernatant was collected for immunocapture and total protein measurement. The total protein was measured by using a micro BCA protein assay kit (Thermo Fisher Scientific) following manufacturer's protocol. The measured concentration levels of S1PR1 were normalized to the total $\mathrm{mg}$ amount of protein in homogenate ( $\mathrm{pg} / \mathrm{mg}$ total protein) to enable direct comparison across samples.

\section{Evaluation of the extraction efficiency of detergent lysis buffer}

Several types of detergent lysis buffer were evaluated and compared for the extraction of S1RP1. PBS buffer was included as well for the purpose of comparison. The extraction buffer and its composition are shown in Table 2. Aliquots of the pulverized human colon were transferred into homogenization tubes in 2 replicates and homogenized in ten volumes of various extraction buffer containing 1\% HALT protease inhibitor cocktail. Following centrifugation at $14,000 \times g$ for $10 \mathrm{~min}$ at $4^{\circ} \mathrm{C}$, the supernatant was collected for tryptic digestion /immunocapture.

\section{Preparations of Ab immobilized magnetic beads}

Prior to sample preparation, anti-peptide antibodies, anti-LTPAQWFLR and anti-SSVSDYVNYDIIVR were covalently immobilized to magnetic tosylactivated beads, respectively. A suspension of $500 \mu$ of magnetic beads was placed in a vial followed by washing 3 times with buffer A (0.1 M borate buffer, $\mathrm{pH} 9.5)$. After the third wash, 
beads were combined with $303 \mu \mathrm{l}$ of $1 \mathrm{mg} / \mathrm{ml}$ antibody in PBS and $151.5 \mu \mathrm{l}$ of buffer C ( $3 \mathrm{M}$ ammonium sulfate in buffer A). The mixture was incubated on a rotator at $37^{\circ} \mathrm{C}$ overnight. The beads were pelleted, and washed twice with $1 \mathrm{ml}$ of glycine buffer $(\mathrm{pH}=2.6)$ to remove potential interference (Ab reagents may contain trace level of antigens), and three-times with $1 \mathrm{ml}$ of buffer D (PBS, $\mathrm{pH} 7.4$ with $0.5 \%[\mathrm{w} / \mathrm{v}]$ bovine serum albumin). The beads were resuspended in $1 \mathrm{ml}$ of buffer $\mathrm{D}$ and incubated on a rotator at $37^{\circ} \mathrm{C}$ for $1 \mathrm{~h}$. After incubation, the beads were washed twice with $1 \mathrm{ml}$ of buffer $\mathrm{E}$ (PBS, $\mathrm{pH} 7.4$ with $0.1 \%$ [w/v] bovine serum albumin) and resuspended in $3 \mathrm{ml}$ of buffer $\mathrm{E}$. The beads were stored at $4^{\circ} \mathrm{C}$.

The antibody immobilized protein G beads were prepared for comparison with tosylactivated beads. AntiLTPAQWFLR and anti-SSVSDYVNYDIIVR antibodies were immobilized to magnetic protein G beads, respectively. A suspension of $1 \mathrm{ml}$ of magnetic protein G beads was placed in a vial followed by washing 3 times with PBST buffer. The beads were resuspended in $1 \mathrm{ml}$ of PBST buffer and combined with $320 \mu \mathrm{g}$ of antibody in PBS. The mixture was incubated on a rotator at RT for $2 \mathrm{~h}$. After incubation, the beads were washed 3 times with $1 \mathrm{ml}$ of PBST buffer containing $1 \%$ bovine serum albumin and resuspended in the same buffer. The beads were stored at $4^{\circ} \mathrm{C}$.

Sample preparation: protein precipitation followed by pellet digestion \& immunocapture

A volume of $100 \mu \mathrm{l}$ of colon tissue extract (supernatant after lysis) was transferred into a 96-well plate (Eppendorf $1 \mathrm{ml}$ Protein LowBind), followed by addition of $400 \mu \mathrm{l}$ of methanol. The samples were vortex-mixed and shaken for $10 \mathrm{~min}$. Following centrifugation at $2000 \mathrm{rpm}$ for $10 \mathrm{~min}$, the supernatants were carefully removed by a Perkin Elmer Janus automated liquid handler. The resulting pellets were resuspended in $500 \mu \mathrm{l}$ of $50 \mathrm{mM}$ ammonium bicarbonate buffer containing $0.05 \%$ zwittergent by vigorous vortex-mixing for $15 \mathrm{~min}$. The samples were then digested with $10 \mu \mathrm{g}$ of trypsin (Promega Trypsin Gold) overnight at $37^{\circ} \mathrm{C}$. After digestion, the samples were incubated at $99^{\circ} \mathrm{C}$ for $30 \mathrm{~min}$ to deactivate trypsin. After the samples were cooled down to room temperature, they were combined with $100 \mu \mathrm{l}$ of PBST and $25 \mu \mathrm{l}$ of the antibody immobilized tosylactivated beads for each peptide, and incubated at RT for $1 \mathrm{~h}$ for anti-peptide immunocapture. The following washing and elution steps were carried out by KingFisher ${ }^{T M}$ Flex System (Thermo Fisher Scientific). The beads were washed three times with $250 \mu \mathrm{l}$ of PBST buffer and one time with $250 \mu \mathrm{l}$ of $50 \mathrm{mM}$ ammonium bicarbonate containing $0.05 \%$ zwittergent. The captured surrogate peptides were eluted from beads by using $100 \mu \mathrm{l}$ of $5 \%$ acetic acid containing $0.05 \%$ zwittergent. An aliquot of $40 \mu \mathrm{l}$ was injected for LC-MS/MS analysis.

\section{Results \& discussion}

Anti-peptide strategy for S1PR1

Due to exceedingly low endogenous levels of S1PR1 and biopsy-sized colon tissue ( $\sim 5 \mathrm{mg}$ colon), a highly sensitive method was required. Immunoaffinity enrichment is the method of choice for sample cleanup to achieve the desired sensitivity. There are some commercially available antibody reagents for S1PR1. Some of these reagents have been tested in our lab for anti-protein immunoaffinity enrichment, but without success. Given the challenges discussed in the introduction section, anti-protein based immunoaffinity enrichment for GPCRs has had limited success. Our assay development strategy involves trypsin digestion followed by peptide immunoaffinity enrichment. The peptides of interest are specifically detected by LC-MS/MS. Rabbit polyclonal antibodies, which were raised against the surrogate peptide sequences, SSVSDYVNYDIIVR and LTPAQWFLR were custom-made for peptide immunoaffinity enrichment. The workflow is shown in Figure 2. Through denaturation, the tightly folded proteins are unfolded so that trypsin can easily access the proteolytic cleavage sites. Proteins are digested into complex mixture of tryptic peptides including the peptides of interest and the high level of peptides from matrix proteins. By immunoaffinity enrichment, the peptides of interest are isolated and the high level of matrix peptides are washed away. The peptides of interest are eluted from beads by acid and then detected by LC-MS/MS.

\section{Selection of surrogate peptides}

Figure 1 shows the structural biology of the S1PR1 receptor. Since seven transmembrane helices are structurally conserved and highly hydrophobic, usually the chance to detect the sequences at these regions by mass spectrometer is small. As a result, the overall sequence coverage is low and this leaves very limited choices for the selection of surrogate peptides. The surrogate peptides are mainly from the extracellular and intracellular segments. Two surrogate peptides, SSVSDYVNYDIIVR and LTPAQWFLR were identified by mass spectrometer. As shown in Figure 1, SSVSDYVNYDIIVR is located at extracellular N-terminus while LTPAQWFLR is mainly from first 


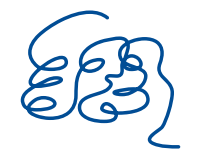

(1)

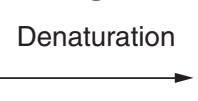

Tightly folded proteins

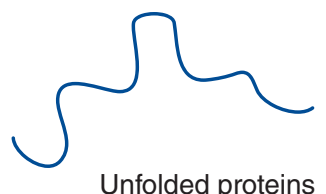

Unfolded proteins
(2)
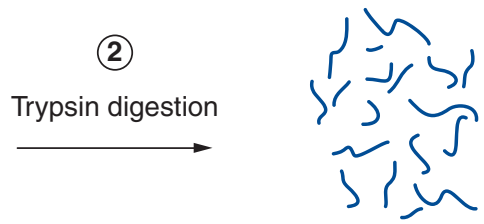

Tryptic peptides from target proteins and matrix proteins

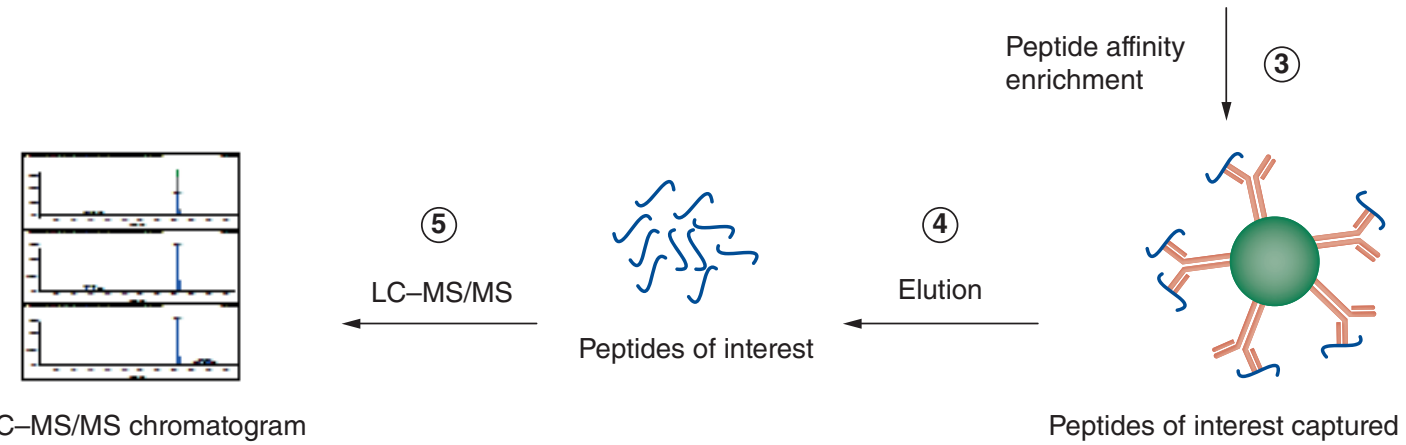

Figure 2. The workflow of peptide immunoaffinity LC-MS/MS for S1PR1 quantitation.

extracellular loop. Table 1 shows MRM transitions and collision energy (CE) for the surrogate peptides and SIL peptides. Two MRM transitions were monitored for each peptide. Since the surrogate peptide LTPAQWFLR with MRM transition $(566.3>917.5)$ provided desired sensitivity for $5 \mathrm{mg}$ colon sample analysis, it was used in the final method for quantitation, while the other peptide and transitions were used for confirmatory purpose.

\section{Extraction \& solubilization of S1PR1 from colon tissue}

Efficient extraction and solubilization of multi-transmembrane proteins from the phospholipid bilayer environment is critical for their quantitation [32]. However, due to their hydrophobic nature, multi-transmembrane proteins are usually difficult to extract and solubilize. Choice of detergents was carefully considered and their extraction efficiency was examined with respect to mass spectrometric response. As shown in Figure 1, seven helices of transmembrane protein pass through the biological membrane, and interact with the hydrophobic tails of the lipid molecules inside of the bilayer, while loops, $\mathrm{N}$-terminus and C-terminus stay in the aqueous environment on either side of the membrane. Due to the similar amphiphilic nature, detergents are commonly used to extract transmembrane proteins by disrupting the phospholipid bilayers and solubilizing membrane proteins. We evaluated several commonly used detergents, including T-PER, 5\% CHAPS, RIPA, 5\% NP-40, Triton X-100 of 1 and 5\%, and 5\% Brij-35, for extraction of endogenous S1PR1 from human colon tissue. PBS buffer was included as well for the purpose of comparison. The extract buffer and its composition are listed in Table 2 . The detailed sample preparation procedure was described in the experiment section. The results are summarized in Figure 3. An increase of Triton X-100 from 1 to $5 \%$, as expected, leads to higher efficiency. The top four detergents are 5\% NP-40, 5\% Triton X-100, T-PER and RIPA.

\section{Optimization of sample preparation}

Considering that endogenous proteins are not identical to recombinant proteins, in this work, we used equal volume aliquots of cynomolgus monkey or human colon tissue homogenate to optimize the sample preparation procedures. Due to hydrophobic nature of S1PR1 and its surrogate peptide, LTPAQWFLR, significant loss of analytes was observed during sample processing. Surfactant, Zwittergent 3-12 detergent (0.05\% in water) was added to stock and working solution, and the most of sample preparation steps to minimize the loss and enhance assay performance and sensitivity.

As shown in the workflow of Figure 2, the first step of sample preparation is protein denaturation. We have evaluated two types of denaturation methods, protein precipitation with methanol and heat denaturation at $99^{\circ} \mathrm{C}$. For the protein precipitation approach, equal volume aliquots of human colon homogenate were precipitated with methanol at ratios of 1:2, 1:3 and 1:4, respectively. The supernatant was removed, and resulting pellets were 

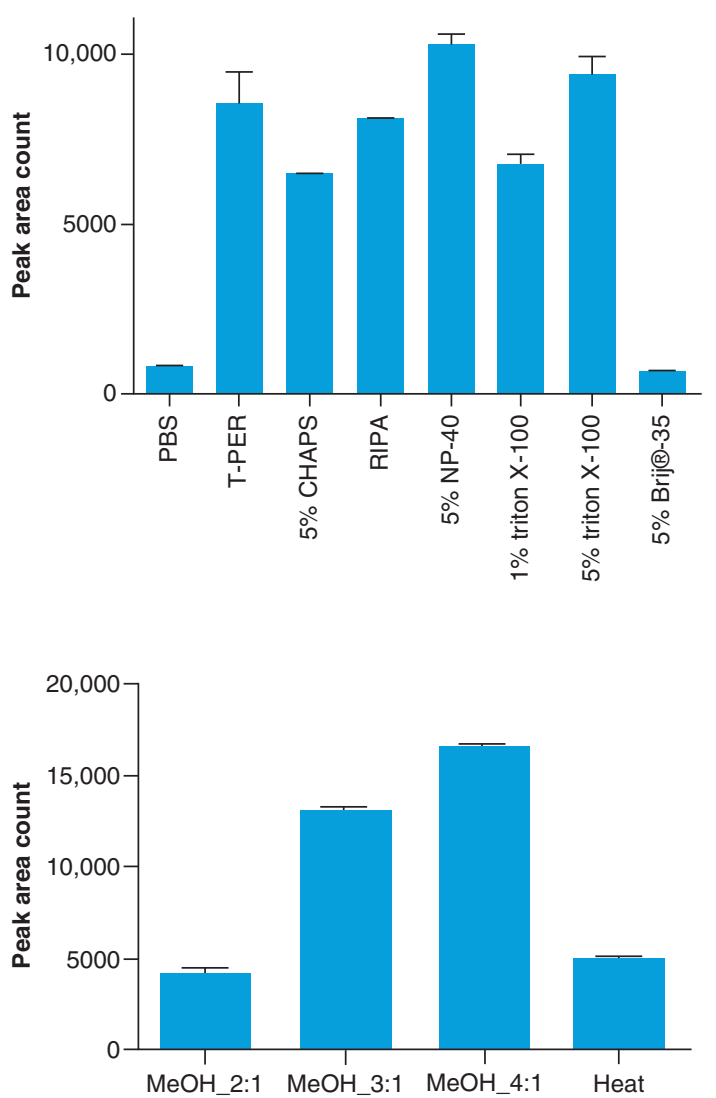

Figure 3. The comparison of LC-MS/MS response of endogenous S1PR1 in human colon tissue by using different extraction buffer. Please see Table 2 for the buffer composition. Peak area count of the surrogate peptide LTPAQWFLR, which is from endogenous S1PR1 in human colon tissue sample.
Figure 4. The effect of denaturation conditions on LC-MS/MS response of the surrogate peptide LTPAQWFLR, which is from endogenous S1PR1 in human colon

homogenate. Protein precipitation denaturation: 2, 3 and 4 volumes of methanol; heat denaturation: $99^{\circ} \mathrm{C}$ for $30 \mathrm{~min}$.

collected and digested. For the heat denaturation, an aliquot of human colon homogenate was mixed with equal volume of $50 \mathrm{mM}$ ammonium bicarbonate buffer and then heated at $99^{\circ} \mathrm{C}$ for $30 \mathrm{~min}$ followed by digestion. The remaining immunocapture steps were same and described in the experiment section. The results (Figure 4) show that 4 volume of methanol gives the highest peak area counts, and thus it was selected as the approach of choice. Protein precipitation does not only denature proteins but also largely removes the detergent and HALT protease inhibitors. As a result, the undesired effects of the detergent and HALT protease inhibitors on digestion and immunoaffinity enrichment in the subsequent steps are reduced. Actually the result is due to a combined effect of denaturation, recovery of immunoaffinity enrichment and digestion efficiency, which will be confirmed in the later discussion.

Pellet digestion was optimized with respect to temperature, time and the amount of trypsin required. For direct digestion of biological samples without first removing significant high-abundance proteins, a large amount of trypsin is required to fully digest all of the proteins, since the total amount of proteins are significant. Bovine pancreatic trypsin is commonly used for this type of applications because it is much more affordable at a large quantity. However, it was observed that the peptides, SIL-SSVSDYVNYDIIVR and SIL-LTPAQWFLR completely disappeared when the neat solution of the SIL-peptides was incubated with the native trypsin even for $10 \mathrm{~min}$ due to a broadened specificity by autolysis. As a result, sequencing grade modified trypsin was required due to its high specific activity and resistance to autolytic digestion. When the sample size was minimized to $5 \mathrm{mg}, 10 \mu \mathrm{g}$ of sequencing grade modified trypsin was sufficient for sample digestion. For the heat denaturation approach, the amount of trypsin required was also evaluated and the results show that much more trypsin was required to fully digest proteins for the samples involving heat denaturation than the samples involving protein precipitation based denaturation. For heat denaturation, HALT protease inhibitor cocktail and T-PER buffer are not removed, and they have inhibitory effect for digestion. HALT protease inhibitor cocktail was added to lysis buffer prior to homogenization of colon tissue samples, to inhibit protease activity and protect cellular proteins from degradation. Its formulation contains six protease inhibitors AEBSF, aprotinin, bestatin, E-64, leupeptin and pepstatin A. Some of them are actually trypsin inhibitors. As a result, more trypsin is needed to fully digest the colon samples. For pellet digestion, two digestion conditions, $37^{\circ} \mathrm{C}$ overnight and $60^{\circ} \mathrm{C}$ for 2 hours, were compared and the results 

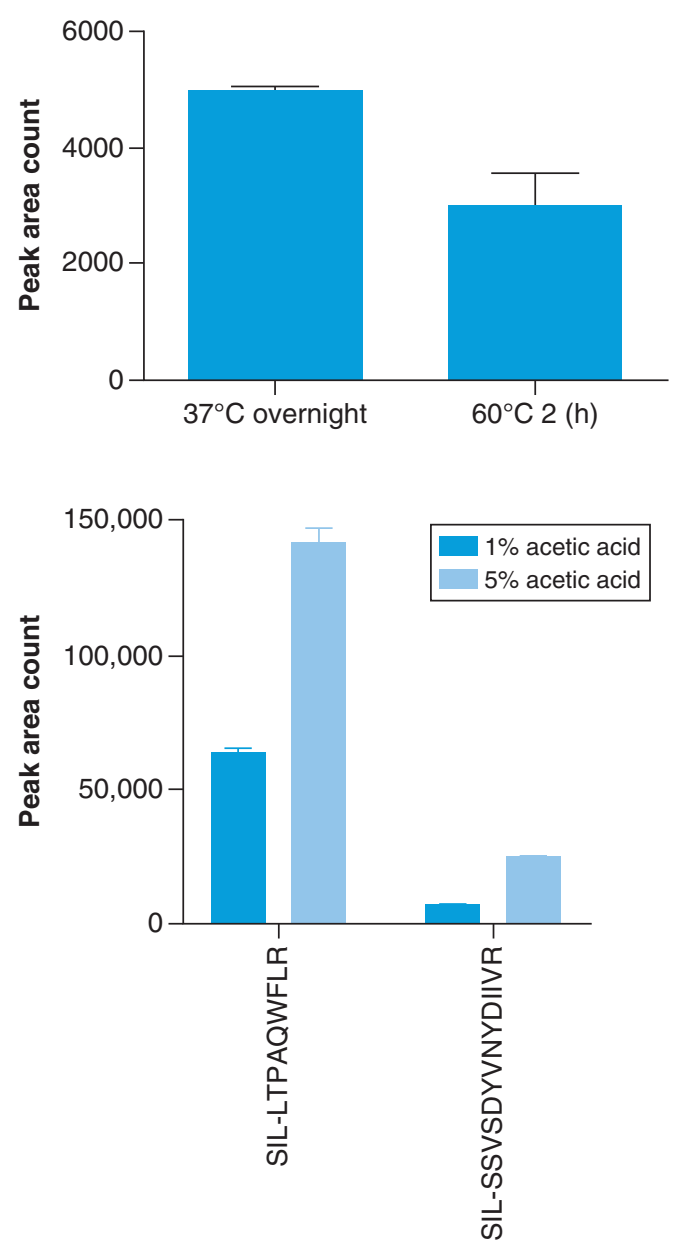

Figure 5. The effect of digestion conditions on LC-MS/MS peak area count of the surrogate peptide LTPAQWFLR, which is from endogenous S1PR1 in human colon homogenate. Trypsin digestion: $37^{\circ} \mathrm{C}$ overnight; $60^{\circ} \mathrm{C}$ for $2 \mathrm{~h}$.
Figure 6. The optimization of the acid elution of the peptides of interest from tosylactivated beads following peptide immunoaffinity enrichment.

are shown in Figure 5. The results show that the overnight digestion at $37^{\circ} \mathrm{C}$ generated higher digestion yield than the digestion at $60^{\circ} \mathrm{C}$ for 2 hours.

Since there are multiple cysteines in S1PR1, reduction and alkylation of disulfide bonds prior to trypsin digestion might make the proteolytic cleavage sites more accessible to trypsin. Digestions with and without reduction/alkylation were evaluated for equal volume aliquots of human colon homogenate samples. Reduction was performed by adding $10 \mu \mathrm{l}$ of $100 \mathrm{mM}$ dithiothreitol in water to the denatured samples followed by incubation at $60^{\circ} \mathrm{C}$ for $15 \mathrm{~min}$. Alkylation was conducted by adding $10 \mu \mathrm{l}$ of $250 \mathrm{mM}$ iodoacetamide in water followed by incubation at RT for 20 min in dark. The following digestion and immunocapture procedures were described in the 'Experimental' section. The results show that there is no difference in yield of the two surrogate peptides. As a result, reduction and alkylation were not included in the final method.

Prior to immunocapture, the samples were heated at $99^{\circ} \mathrm{C}$ for $30 \mathrm{~min}$ to deactivate trypsin, since trypsin has inhibitory effect on immunocapture. For beads based immunoaffinity enrichment, both protein $\mathrm{G}$ beads and tosylactivated beads were evaluated and compared. Tosylactivated beads offered cleaner MRM background noise than protein $G$ beads and was thus used in the final method. A volume of $25 \mu \mathrm{l}$ of tosylactivated bead suspension, which could be immobilized with $0.125-1.25 \mu \mathrm{g}$ antibody, provides sufficient binding capacity for immunoaffinity enrichment of the peptides of interest in $100 \mu \mathrm{l}$ of the samples. The acid elution of the peptides of interest from tosylactivated beads was optimized. Both SIL-LTPAQWFLR and SIL-SSVSDYVNYDIIVR were spiked into PBS buffer. Following peptide immunoaffinity enrichment, acetic acid (1 and 5\%) with 0.05\% zwittergent were used to elute the peptides of interest. The peak area counts were compared and results are shown in Figure 6. The results show the peak area counts of the peptides with 5\% acetic acid elution are 2-3.5 times higher than the peak area counts with $1 \%$ acetic acid elution. For immunoaffinity enrichment, we also evaluated and compared the effects of detergents on immunocapture recovery by using SIL peptides. Both SIL-LTPAQWFLR and SIL-SSVSDYVNYDIIVR were spiked into three matrices, PBS buffer, T-PER based cynomolgus monkey colon 
Table 3. The effects of detergents on immunocapture recovery for SIL-LTPAQWFLR and SIL-SSVSDYVNYDIIVR $(1 \mathrm{nM})$ in duplicates.

\begin{tabular}{|lll|}
\hline Sample matrix & Peak area count of SIL-LTPAQWFLR & $\begin{array}{c}\text { Peak area count of } \\
\text { SIL-SSVSDYVNYDIIVR }\end{array}$ \\
\hline PBS buffer_1 & 137,651 & 25,377 \\
\hline PBS buffer_2 & 145,841 & 25,477 \\
\hline Cyno colon homogenate with T-per_1 & 106,934 & 3024 \\
\hline Cyno colon homogenate with T-per_2 & 116,838 & 3626 \\
\hline Cyno colon homogenate with RIPA_1 & 18,625 & 311 \\
\hline Cyno colon homogenate with RIPA_2 & 10,561 & 336 \\
\hline
\end{tabular}

\begin{tabular}{|c|c|c|c|}
\hline S1P1R1_HUMAN & 60 & ILENIFVLLTIWKTKKFHRPMYYFIGNLALSDLLAGVAYTANLLLSGATTYK. LTPAQWFLR.EGSMFVALS & 129 \\
\hline S1P1R1_MOUSE & 60 & ILENIFVLLTIWKTKKFHRPMYYFIGNLALSDLLAGVAYTANLLLSGATTYK. LTPAQWFLR.EGSMFVALS & 129 \\
\hline S1P1R1_RAT & 61 & ILENIFVLLTIWKTKKFHRPMYYFIGNLALSDLLAGVAYTANLLLSGATTYK. LTPAQWFLR.EGSMFVALS & 130 \\
\hline S1P1R1_PIG & 60 & ILENIFVLLTIWKTKKFHRPMYYFIGNLALSDLLAGVAYIANLLLSGATTYK. I & 9 \\
\hline S1P1R1_CYNO & 60 & ILENIFVLLTIWKTKKFHRPMYYFIGNLALSDLLAGVAYTANLLLSGATTYK. LTPAQV & \\
\hline S1P1R1_DOG & 60 & ILENIFVLLTIWKTKKFHRPMYYFIGNLALSDLLAGVAYTANLLLSGATTYK. & \\
\hline
\end{tabular}

Figure 7. Alignment of S1PR1 receptor protein sequences for human, mouse, rat, pig, cynomolgus monkey and dog.

homogenate and RIPA based cynomolgus monkey colon homogenate. Samples were processed by immunoaffinity enrichment as described in the 'Experimental' section. The peak area counts were compared and results are shown in Table 3. T-PER is mild detergent while RIPA is a strong detergent. For SIL-LTPAQWFLR, immunocapture recovery is good for T-PER based colon homogenate while significant loss was observed for RIPA based colon homogenate. For SIL-SSVSDYVNYDIIVR, significant loss was observed for both T-PER and RIPA-based colon homogenate. The results suggest that the removal of detergents by using protein precipitation based denaturation is necessary for the subsequent immunocapture.

\section{Assay performance}

Due to the endogenous level of biomarkers in authentic matrices, surrogate matrices such as buffer or a corresponding matrix from another species, which do not contain the analytes of interest are commonly used for calibration standards. Since the surrogate peptide, LTPAQWFLR is a common peptide across multiple species including mouse, rat, pig, cynomolgus monkey and dog as shown in Figure 7, colon tissues from these species can't be used as matrix for calibration standards. As a result, the lysis buffer with $1 \%$ HALT protease inhibitor cocktail and $0.1 \%$ BSA was chosen as surrogate matrix for calibration standards. Two sets of calibration standards with the recombinant S1PR1 protein and SIL peptide were assessed to address any potential discrepancies. For the first set of calibration standards, human S1PR1 full-length recombinant protein without tag, was spiked and processed following the same procedure as colon samples. For the second set, the SIL peptides were spiked and prepared by peptide immunoaffinity enrichment procedure. These two sets of duplicate calibration standards, which were placed at the beginning and end of the analytical run, were analyzed along with 30 human colon samples. Figure 8 shows the calibration curves of human S1PR1 full-length recombinant protein and SIL peptide. It demonstrates that two curves are well matched, indicating that protein digestion is complete. As a result, the percentage difference of the measured S1PR1 concentration levels between protein curve and peptide curve is within $5.8 \%$ for all 30 colon samples. The recombinant proteins are commercially available only for human S1PR1 but not for other species such as mouse, rat, pig, cynomolgus monkey and dog. Therefore, peptide calibration curves need to be used to quantify S1PR1 in other species. Furthermore given that the difficult and high cost associated with generation of multi-transmembrane recombinant proteins, using a peptide calibration standards leads to significant time and cost savings.

The assay performance was further evaluated by immunocapture recovery, matrix effect, precision and accuracy. The average recovery of $91 \%$ was determined by comparing the peak area of SIL-LTPAQWFLR between a sample which was spiked prior to immunocapture, and a sample, which was spiked after immunocapture. The matrix effect 


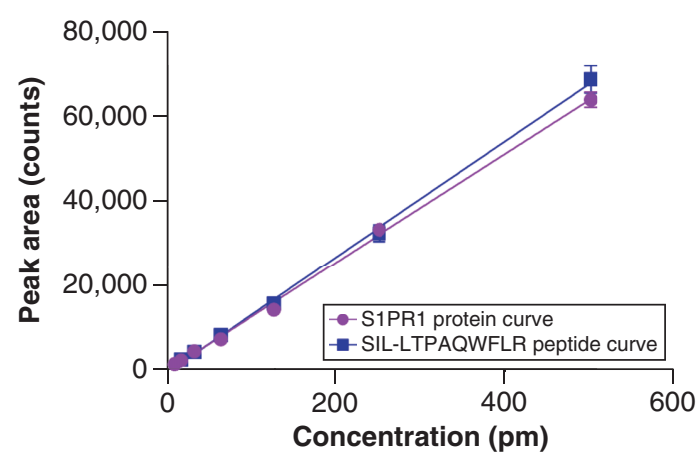

Figure 8. Calibration curves of human S1PR1 full-length recombinant protein and SIL-LTPAQWFLR. Calibration curve range is from 7.8 to $500 \mathrm{pM}$.

\begin{tabular}{|c|c|c|c|c|c|c|}
\hline \multirow[t]{2}{*}{ Statistic } & \multicolumn{6}{|c|}{ Concentration (pM) } \\
\hline & 15.63 & 31.25 & 62.5 & 125 & 250 & 500 \\
\hline \multicolumn{7}{|c|}{ Quality controls of S1PR1 in 3 replicates } \\
\hline$\%$ DEV & $3 \%$ & $4 \%$ & $-9 \%$ & $-3 \%$ & $7 \%$ & $2 \%$ \\
\hline$\% \mathrm{CV}$ & $7 \%$ & $9 \%$ & $5 \%$ & $5 \%$ & $3 \%$ & $5 \%$ \\
\hline \multicolumn{7}{|c|}{ Quality controls of SIL-LTPAQWFLR in 3 replicates } \\
\hline$\%$ DEV & $-2 \%$ & $-6 \%$ & $2 \%$ & $-1 \%$ & $5 \%$ & $6 \%$ \\
\hline$\% \mathrm{CV}$ & $15 \%$ & $9 \%$ & $5 \%$ & $5 \%$ & $5 \%$ & $4 \%$ \\
\hline
\end{tabular}

was determined by comparing the peak area of SIL-LTPAQWFLR between a postspiked sample, which was spiked after digestion/immunocapture of colon sample and a neat SIL-LTPAQWFLR sample. The average matrix effect is $87 \%$. The LLOQ of the assay is $7.8 \mathrm{pM}$ and the blank is clean without interference peaks. The calibration curve range is from 7.8 to $500 \mathrm{pM}$. A linear least-squares regression with weighting factor of $\left(1 / \mathrm{x}^{2}\right)$ was used [33], and the regression coefficient of the calibration curve was 0.993 for the protein curve and 0.996 for the peptide curve. The accuracy and precision of QCs for both S1PR1 protein and SIL peptide are shown in Table 4. The accuracy and precision of QCs for the protein are within \pm 9 and $9 \%$, respectively, and the accuracy and precision of QCs for SIL peptide are within $\pm 6 \%$ and $15 \%$, respectively. The within-run variability for unknown samples were assessed for 3 unknown human colon tissue samples. Each human colon tissue sample $(\sim 200 \mathrm{mg})$ was pulverized, and three aliquots of each pulverized sample were processed and analyzed by LC-MS/MS. The within-run variability of the measured concentration levels for three aliquots are 1, 3 and $16 \%$, for three unknown samples respectively.

LC-MS/MS extracted ion chromatograms of human S1PR1 in $\sim 5 \mathrm{mg}$ human colon samples are shown in Figure 9. The surrogate peptide LTPAQWFLR with the $566.3>917.5$ MRM transition was used for quantitation, and the other peptide and MRM transitions were used for confirmation and specificity. Basal S1PR1 levels for 10 normal human colon samples $(\sim 5 \mathrm{mg})$ were measured and the data are shown in Figure 10. Total protein concentration levels were determined for all colon tissue homogenate and the concentration levels of S1PR1 were normalized by the corresponding total protein concentration. This is an essential step for actual biopsy studies as the expected samples will be too small in size to accurately weight out and without a proper normalization the data cannot be properly interpreted. Normalization of tissue protein levels to total protein is a well-established approach.

High sequence homology of S1PR1 was observed between human and other species including cynomolgus monkey, rat, mouse, dog and pig as shown in Figure 7. LTPAQWFLR is a common surrogate peptide across all these species. Therefore, the method for human S1PR1 could be applied for quantitative determination of S1PR1 in other 5 species. It has been successfully applied for analysis of cynomolgus monkey colon tissue samples. It could potentially allow for direct comparison of S1PR1 expression levels among different species.

Since the colon tissue samples were extracted and solubilized by detergents without first isolating cell surface S1PR1 from membrane, this assay is considered for measurement of total S1PR1 levels, which is total of receptors in the membrane and receptors that have been internalized. Isolation of cell surface S1PR1 prior to detergent-based extraction is required in order to measure cell surface S1PR1 by peptide immunoaffinity LC-MS/MS. 

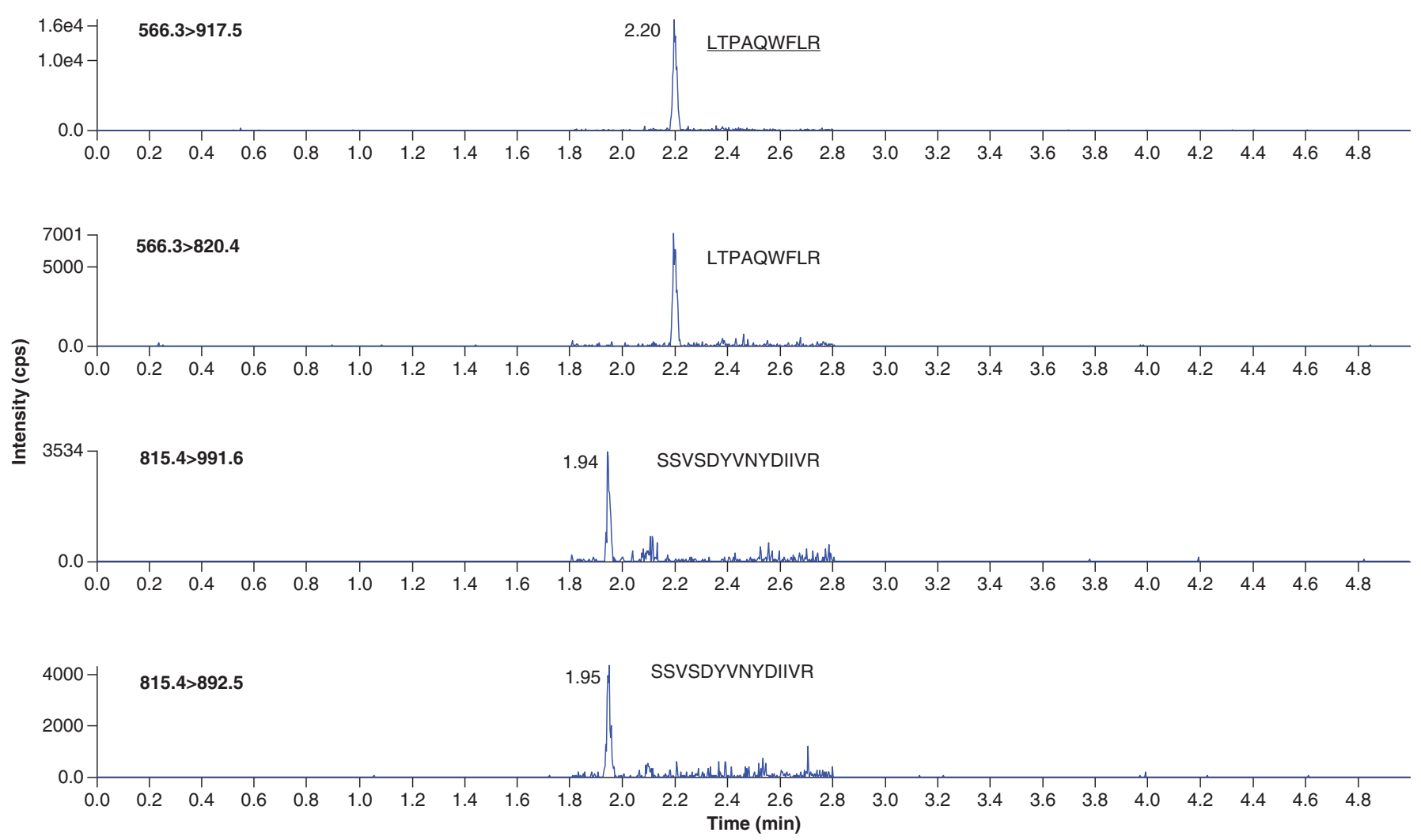

Figure 9. LC-MS/MS extracted ion chromatograms of human S1PR1 in $\sim \mathbf{5}$ mg human colon samples. The surrogate peptide LTPAQWFLR with the $566.3>917.5$ MRM transition was used for quantitation. The other peptide and MRM transitions monitored for specificity.

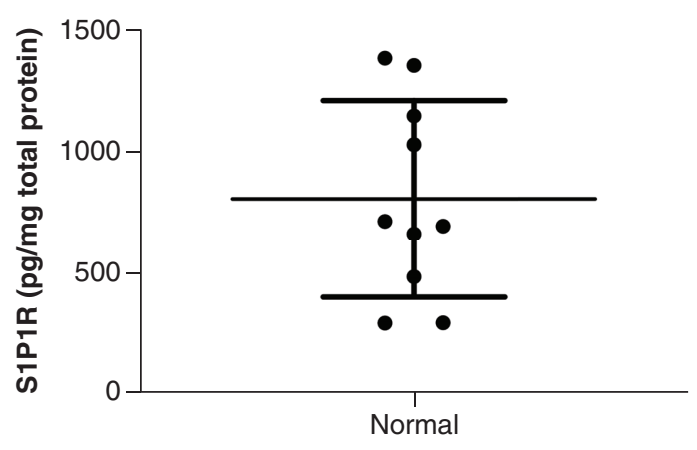

Figure 10. Basal S1PR1 levels for 10 normal human colon samples ( $5 \mathrm{mg}$ ).

\section{Conclusion}

This is the first report of quantitative determination of the GPCR protein, S1PR1 by using peptide immunoaffinity LC-MS/MS. It covers bioanalytical considerations and strategies for GPCR protein quantitation by using LCMS/MS based methods, including extraction, immunoaffinity enrichment, low sequence coverage and choice of calibrants. Several detergents have been evaluated for extraction of S1PR1 from colon tissue samples. Peptide immunoaffinity enrichment has been employed to specifically isolate the surrogate peptides with high recovery and low matrix effect. It is also demonstrated that with the optimized conditions, protein and peptide calibration curves are well matched and provide the same results for S1PR1 quantitation. A sensitive LC-MS/MS assay with an LLOQ of 7.8 pM has been achieved and the basal S1PR1 levels in small biopsy-sized human colon tissue samples have been determined. Since all the GPCR proteins share the similar structures and challenges, the same strategy and approach have been successfully applied to several other GPCR protein quantitation in our laboratory. 


\section{Future perspective}

GPCRs represents one of the most important therapeutic target class because of their key roles in transmitting chemical signals into a wide array of different cells and their clinical relevance for a wide range of diseases. However, due to the intrinsic nature of GPCR proteins, the characterizations and quantitative measurements of GPCR proteins are challenging and therefore a lot less is known and published as compared with other proteins. Their intrinsic nature makes it particularly challenging to generate specific antibody reagents for protein immunoaffinity enrichment. In this work, we employed a peptide immunoaffinity LC-MS/MS approach for specifically enriching and detecting surrogate peptides. Our work demonstrates that with optimization, peptide immunoaffinity LCMS/MS methods can achieve the desired sensitivity for the low abundance GPCR protein, S1PR1. The same strategy could be applied to other GPCR proteins.

A number of bioanalytical challenges and considerations have been addressed, and the assay has been fully optimized to ensure that the assay can reliably measure what intend to be measured and generate meaningful data for the discovery studies. As the assay qualification strategy for early exploratory biomarkers, full validation is not required. However several critical checks have been assessed as part of fit-for-purpose qualification to ensure that the bioanalytical method rigor is suitable for the intended use of the biomarker data in drug discovery. To further improve assay quality and address the issues for matrix difference between samples and calibration standards, insample calibration curve using multiple isotopologue reaction monitoring (MIRM) of a stable isotopically labeled analyte will be evaluated and applied for future studies [34,35]. It eliminates the issue of lacking of analyte-free authentic matrices for biomarker quantitation, while keeps samples and calibration standards in the exact same matrices.

The surrogate peptide, LTPAQWFLR is a common peptide in human, cynomolgus monkey, rat, mouse, dog and pig. As a result, the anti-peptide antibodies and LC-MS/MS method for human S1PR1 can be applied for multiple species. Preclinical studies rely heavily on animal models of human disease to assess efficacy and safety risk, and, therefore, biomarker assays are often required for multiple species. Peptide immunoaffinity LC-MS/MS could be one-size-fit-all approach for multiple species in many cases. Another advantage of peptide immunoaffinity enrichment is that it can be applied to formalin-fixed paraffin-embedded (FFPE) tissue samples in pre-clinical or clinical studies where anti-protein based immunoaffinity approach is unlikely to work. It could also be a potential method for measuring total ligand following dosing with the anti-ligand antibody drugs. Peptide immunoaffinity enrichment bypasses issues around generation of a non-inhibitory anti-ligand antibody as capture reagent, which

\section{Executive summary}

\section{Background}

- The G protein-coupled receptors (GPCRs) are important drug targets and account for more than a third of approved drugs covering wide range of disease types, while numerous novel GPCRs are being studied for the unmet medical needs. Quantitative determination of GPCR expression is thus important for drug discovery and development.

- Due to their hydrophobic nature and intrinsic difficulties of extraction and solubilization, along with low expression levels, quantitative determination of GPCR proteins remains challenging.

- Since all the GPCR proteins share the similar structures, it is important to understand challenges and establish bioanalytical strategies for assay development.

- Due to extremely low abundance and small biopsy-sized colon tissue, a highly sensitive method was required to quantify S1PR1.

Results

- Developed bioanalytical strategies for absolute quantitation of the GPCR protein, S1PR1 in colon tissue samples.

- Developed a highly sensitive peptide immunoaffinity LC-MS/MS method for S1PR1 with LLOQ of 7.81 pM.

- The assay was employed for quantitative determination of endogenous basal levels of S1PR1 in $\sim 5 \mathrm{mg}$ human colon tissue samples.

Conclusion

- This is the first report of quantitative determination of S1PR1 in biological matrix by using peptide immunoaffinity LC-MS/MS.

- The assay could be used to quantify S1PR1 in multiple species, since a common peptide is used.

- It is demonstrated that with optimization, peptide immunoaffinity LC-MS methods could achieve the desired sensitivity for the low abundance GPCR protein, S1PR1.

- The same strategy could be applied to other GPCR protein quantitation. 
should not compete with drug (antibody) and detection antibody [17]. This could significantly save on time and cost involved in screening for antibody reagents. Peptide immunoaffinity LC-MS/MS is a complementary strategy to anti-protein immunoaffinity assays and is being increasingly used for protein quantitation.

Financial \& competing interests disclosure

The authors of this article are current or past employees of Bristol-Myers Squibb Company. All financial support for the studies reported herein was provided by Bristol Myers Squibb Company. The authors have no other relevant affiliations or financial involvement with any organization or entity with a financial interest in or financial conflict with the subject matter or materials discussed in the manuscript apart from those disclosed.

No writing assistance was utilized in the production of this manuscript.

\section{References}

Papers of special note have been highlighted as: $\bullet$ of interest; $\bullet \bullet$ of considerable interest

1. Hanson MA, Roth CB, Jo E et al. Crystal Structure of a lipid G protein-coupled receptor. Science 335(6070), 851-855 (2012).

2. Gatfield J, Monnier L, Studer R, Bolli MH, Steiner B, Nayler O. Sphingosine-1-phosphate (S1P) displays sustained S1P1 receptor agonism and signaling through S1P lyase-dependent receptor recycling. Cell. Signal. 26(7), 1576-1588 (2014).

3. Karuppuchamy T, Behrens EH, González-Cabrera P et al. Sphingosine-1-phosphate receptor-1 (S1P1) is expressed by lymphocytes, dendritic cells, and endothelium and modulated during inflammatory bowel disease. Mucosal Immunol. 10(1), 162-171 (2017).

4. Dyckman AJ. Modulators of sphingosine-1-phosphate pathway biology: recent advances of sphingosine-1-phosphate receptor 1 (S1P1) agonists and future perspectives. J. Med. Chem. 60(13), 5267-5289 (2017).

5. Gonzalez-Cabrera PJ, Brown S, Studer SM, Rosen H. S1P signaling: new therapies and opportunities. F1000Prime Rep. 6, 109-109 (2014).

6. Chun J, Hartung HP. Mechanism of action of oral fingolimod (FTY720) in multiple sclerosis. Clin. Neuropharmacol. 33(2), 91-101 (2010).

7. Rosenbaum DM, Rasmussen SGF, Kobilka BK. The structure and function of G-protein-coupled receptors. Nature 459(7245), 356-363 (2009).

8. Jo M, Jung ST. Engineering therapeutic antibodies targeting G-protein-coupled receptors. Exp. Mol. Med. 48 , e207 (2016).

9. Hauser AS, Attwood MM, Rask-Andersen M, Schioth HB, Gloriam DE. Trends in GPCR drug discovery: new agents, targets and indications. Nat. Rev. Drug Discov. 16(12), 829-842 (2017).

- Covers the importance of G protein-coupled receptor (GPCR) therapeutic targets in clinical trials, and emerging trends and opportunities in GPCR drug discovery.

10. Usman S, Khawer M, Rafique S, Naz Z, Saleem K. The current status of anti GPCR drugs against different cancers. J. Pharm. Anal. doi:https://doi.org/10.1016/j.jpha.2020.01.001 (2020).

11. Sriram K, Wiley SZ, Moyung K et al. Detection and quantification of GPCR mRNA: an assessment and implications of data from high-content methods. bioRxiv doi:10.1101/734863 734863 (2019).

12. Peeters MC, Van Westen GJ, Li Q, Ap IJ. Importance of the extracellular loops in G protein-coupled receptors for ligand recognition and receptor activation. Trends Pharmacol. Sci. 32(1), 35-42 (2011).

13. Michel MC, Wieland T, Tsujimoto G. How reliable are G-protein-coupled receptor antibodies? Naunyn-Schmiedeberg's Arch. Pharmacol. 379(4), 385-388 (2009).

- Highlights the specificity issues with GPCR antibodies, and considerations for antibody validation to ensure a selective and reliable assay for measurement of GPCRs.

14. Ackermann BL. Immunoaffinity MS: adding increased value through hybrid methods. Bioanalysis 8(15), 1535-1537 (2016).

15. Kang L, Weng N, Jian W. LC-MS bioanalysis of intact proteins and peptides. Biomed. Chromatogr. 34(1), e4633 (2020).

16. Hongwei Z, Qing X, Baomin X et al. Development of a highly sensitive liquid chromatography/tandem mass spectrometry method to quantify total and free levels of a target protein, interferon-gamma-inducible protein-10, at picomolar levels in human serum. Rapid Commun. Mass Spectrom. 28(13), 1535-1543 (2014).

17. Zhang H, Gu H, Shipkova P et al. Immunoaffinity LC-MS/MS for quantitative determination of a free and total protein target as a target engagement biomarker. Bioanalysis 9(20), 1573-1588 (2017).

18. Neubert H, Shuford CM, Olah TV et al. Protein biomarker quantification by immunoaffinity liquid chromatography-tandem mass spectrometry: current state and future vision. Clin. Chem. 66(2), 282-301 (2020).

- This review article covers the advantages, applications and opportunities for immunoaffinity enrichment LC-MS based assays for protein biomarker quantification.

19. Stanley Z, Wenying J. Recent advances in absolute quantification of peptides and proteins using LC-MS. Rev. Anal. Chem. 33(1), 31-47 (2014). 
20. Neubert H, Muirhead D, Kabir M, Grace C, Cleton A, Arends R. Sequential protein and peptide immunoaffinity capture for mass spectrometry-based quantification of total human $\beta$-nerve growth factor. Anal. Chem. 85(3), 1719-1726 (2013).

21. Levernæs MCS, Farhat B, Oulie I et al. Immunocapture sample clean-up in determination of low abundant protein biomarkers - a feasibility study of peptide capture by anti-protein antibodies. RSC Adv. 9(60), 34902-34911 (2019).

22. Hoofnagle AN, Becker JO, Wener MH, Heinecke JW. Quantification of thyroglobulin, a low-abundance serum protein, by immunoaffinity peptide enrichment and tandem mass spectrometry. Clin. Chem. 54(11), 1796-1804 (2008).

23. Ackermann BL, Berna MJ. Coupling immunoaffinity techniques with MS for quantitative analysis of low-abundance protein biomarkers. Expert Rev. Proteomics 4(2), 175-186 (2007).

24. Fan Y-Y, Neubert H. Quantitative analysis of human neonatal $F_{c}$ receptor $\left(F_{c} R n\right)$ tissue expression in transgenic mice by online peptide immuno-affinity LC-HRMS. Anal. Chem. 88(8), 4239-4247 (2016).

25. Palandra J, Finelli A, Zhu M, Masferrer J, Neubert H. Highly specific and sensitive measurements of human and monkey interleukin 21 using sequential protein and tryptic peptide immunoaffinity LC-MS/MS. Anal. Chem. 85(11), 5522-5529 (2013).

26. Kushnir MM, Rockwood AL, Roberts WL, Abraham D, Hoofnagle AN, Meikle AW. Measurement of thyroglobulin by liquid chromatography-tandem mass spectrometry in serum and plasma in the presence of antithyroglobulin autoantibodies. Clin. Chem. 59(6), 982-990 (2013).

27. Becker JO, Hoofnagle AN. Replacing immunoassays with tryptic digestion-peptide immunoaffinity enrichment and LC-MS/MS. Bioanalysis 4(3), 281-290 (2012).

28. Schultz GA, Mccardle K, Neubert H. Large-scale implementation of sequential protein and peptide immunoaffinity enrichment LC/nanoLC-MS/MS for human $\beta$-nerve growth factor. Bioanalysis 8(8), 753-764 (2016).

29. Anderson NL, Anderson NG, Haines LR, Hardie DB, Olafson RW, Pearson TW. Mass spectrometric quantitation of peptides and proteins using stable isotope standards and capture by anti-peptide antibodies (SISCAPA). J. Proteome Res. 3(2), 235-244 (2004).

- The first report of peptide immunoaffinity LC-MS/MS assays, also termed stable isotope standards and capture by anti-peptide antibodies (SISCAPA).

30. Whiteaker JR, Paulovich AG. Peptide immunoaffinity enrichment coupled with mass spectrometry for peptide and protein quantification. Clin. Lab. Med. 31(3), 385-396 (2011).

31. Whiteaker JR, Zhao L, Yan P et al. Peptide immunoaffinity enrichment and targeted mass spectrometry enables multiplex, quantitative pharmacodynamic studies of phospho-signaling. Mol. Cell. Proteomics 14(8), 2261 (2015).

32. Arachea BT, Sun Z, Potente N, Malik R, Isailovic D, Viola RE. Detergent selection for enhanced extraction of membrane proteins. Protein Exp. Purif. 86(1), 12-20 (2012).

33. Gu H, Liu G, Wang J, Aubry A-F, Arnold ME. Selecting the correct weighting factors for linear and quadratic calibration curves with least-squares regression algorithm in bioanalytical LC-MS/MS assays and impacts of using incorrect weighting factors on curve stability, data quality, and assay performance. Anal. Chem. 86(18), 8959-8966 (2014).

34. Gu H, Zhao Y, Demichele M et al. In-sample calibration curve using multiple isotopologue reaction monitoring of a stable isotopically labeled analyte for instant LC-MS/MS bioanalysis and quantitative proteomics. Anal. Chem. 91(3), 2536-2543 (2019).

-• For the first time, this paper introduces a novel MIRM-ISCC-LC-MS/MS methodology for instant, accurate and reliable LC-MS/MS bioanalysis of biomarkers without using external calibration curves. It eliminates the issue of lacking of analyte-free authentic matrices for biomarker quantitation, while keeps samples and calibration standards in the exact same matrices.

35. Gu H, Zhao Y, Demichele M et al. Eliminating preparation of multisample external calibration curves and dilution of study samples using the multiple isotopologue reaction monitoring (MIRM) technique in quantitative LC-MS/MS bioanalysis. Anal. Chem. 91(13), 8652-8659 (2019). 